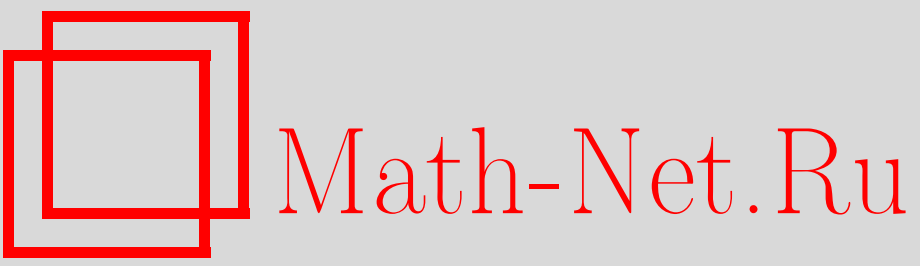

О. Б. Цехан, Декомпозиция сингулярно возмущенной функциональнодифференциальной системы на основе невырожденного преобразования, Итоги науки и техн. Сер. Соврем. мат. и ее прил. Темат. обз., 2021, том 190, 130-143

DOI: https://doi.org/10.36535/0233-6723-2021-190-130-143

Использование Общероссийского математического портала Math-Net.Ru подразумевает, что вы прочитали и согласны с пользовательским соглашением

http://www.mathnet.ru/rus/agreement

Параметры загрузки:

IP : 54.147 .182 .235

26 апреля 2023 г., 15:22:28 


\title{
ДЕКОМПОЗИЦИЯ СИНГУЛЯРНО ВОЗМУЩЕННОЙ ФУНКЦИОНАЛЬНО-ДИФФЕРЕНЦИАЛЬНОЙ СИСТЕМЫ НА ОСНОВЕ НЕВЫРОЖДЕННОГО ПРЕОБРАЗОВАНИЯ
}

\author{
(c) 2021 г. $\quad$ О. Б. ЦЕХАН
}

\begin{abstract}
АннотАция. Для линейной стационарной сингулярно возмущенной функционально-дифференциальной системы управления с малым параметром при старшей производной и с конечным запаздыванием в медленных переменных состояния обоснована декомпозиция с помощью невырожденного преобразования переменных, обобщающего известное преобразование типа Chang. Преобразование выполняет расщепление исходной двухтемповой системы на две независимые подсистемы меньшей размерности: отдельно относительно быстрых и медленных переменных. Доказано, что расщепляющее преобразование может быть построено с любой точностью аппроксимации в виде асимптотического разложения по степеням малого параметра, указана итеративная схема нахождения членов асимптотического ряда. Получена оценка значений параметра, при которой справедлива аппроксимация. На основании построенной декомпозиции установлено, что при достаточно малых значениях параметра спектр системы разделяется на два множества: отдельно с «малыми» и «большими» собственными значениями. Приведены примеры построения аппроксимаций преобразования.
\end{abstract}

Ключевые слова: сингулярно возмущенная система, функционально-дифференциальная система, запаздывание, расщепляющее преобразование, декомпозиция.

\section{DECOMPOSITION OF A SINGULARLY PERTURBED FUNCTIONAL-DIFFERENTIAL SYSTEM BASED ON A NONDEGENERATE TRANSFORMATION}

\author{
(c) 2021 O. B. TSEKHAN
}

\begin{abstract}
For a linear, stationary, singularly perturbed functional-differential control system with a small coefficient of the highest derivative and a finite delay in slow state variables, we justify the decomposition by a nondegenerate transformation of variables, which is a generalization of the well-known Chang transformation. This transformation splits the original two-rate system into two independent subsystems of lower dimension separately for the fast and slow variables. We prove that the splitting transformation can be constructed with any approximation accuracy in the form of an asymptotic expansion in powers of a small parameter and propose an iterative scheme for calculating the asymptotic series. Based on the decomposition constructed, we establish that for sufficiently small values ??of the parameter, the spectrum of the system is split into two sets, separately for "small" and "large" eigenvalues. Also, we give examples of constructing transform approximations.
\end{abstract}

Keywords and phrases: singularly perturbed system, functional-differential system, delay, splitting transformation, decomposition.

AMS Subject Classification: 34K06, 34K17, 34K26, 34K 35

Работа выполнена при поддержке Министерства образования Республики Беларусь в рамках государственной программы научных исследований Республики Беларусь «Конвергенция-2020» (шифр задания 1.3.02). 
1. Введение. Системы дифференциальных уравнений с малым параметром при старших производных относятся к классу сингулярно возмущенных систем и являются математическими моделями динамических систем, в которых реализуется несколько взаимосвязанных подпроцессов с существенно различающимися темпами. Интерес к сингулярно возмущенным системам вызван потребностями практики в связи с интенсивным развитием таких областей, как теория автоматического регулирования, теория нелинейных колебаний, квантовая механика, газодинамика, биология, химическая кинетика и другие (см., например, ссылки в обзорных статьях $[1,2,19]$. В указанных обзорах можно найти также ссылки на множество публикаций, посвященных вопросам анализа и синтеза сингулярно возмущенных систем.

В технике, экономике, технологиях, биологии, экологии, социальной сфере и т. п. (см., например, ссылки в [15]) запаздывание сопровождает различные процессы, связанные с передачей массы, энергии, информации и т. п. Наличие запаздывания отражает объективное свойство инерционности систем, в той или иной мере присущее любым реальным процессам, проявляется в виде зависимости текущей скорости изменения выходных переменных системы от их значений в предшествующие моменты времени и приводит к моделям, которые описываются функциональнодифференциальными уравнениями запаздывающего типа (см. [6]).

При решении задач анализа и управления сложными объектами часто возникают проблемы, обусловленные высокой размерностью моделей и наличием разнотемповых переменных. В связи с этим актуальной является проблема декомпозиции - расчленения моделей большого порядка на несвязные или слабосвязные модели меньшей размерности. Применение методов декомпозиции для анализа и синтеза сложных систем позволяет упрощать и распараллеливать алгоритмы, обеспечивает возможность управления различными частями систем. Декомпозиция может строиться на различных принципах. Применительно к системам с разнотемповыми движениями возможна декомпозиция исходной сингулярно возмущенной системы на подсистемы меньшей размерности с разделенными по темпам переменными.

Для линейных сингулярно возмущенных систем декомпозицию можно реализовать с помощью невырожденной замены переменных, осуществляющей эквивалентный переход от сингулярно возмущенной системы к системе с разделенными по скоростям движениями. Впервые невырожденное линейное преобразование для нестационарной линейной сингулярно возмущенной системы без запаздывания с малым параметром при старших производных части переменных предложено в [7] и применено для полного расщепления исходной системы с быстрыми и медленными переменными на две независимые подсистемы в [12,13]. Матрицы, участвующие в формировании преобразования Chang, являются решениями алгебраических уравнений Риккати и Сильвестра. Их нахождение является ключевым шагом в построении этого преобразования. В [13] построена аппроксимация первого порядка решения соответствующего уравнения Риккати с помощью ряда Маклорена. Там же отмечается, что получение аппроксимации более высокого порядка является механическим повторением случая аппроксимации первого порядка.

Обобщение преобразования Chang для декомпозиции линейных сингулярно возмущенных медленно изменяющихся во времени систем предложено в $[17,18]$. В [18] доказана теорема о разложении в ряды Маклорена матричных компонент линейного преобразования и об итерационном нахождении элементов $k$-го порядка аппроксимации этих рядов. Основываясь на итерационном решении, предложено понятие аппроксимации $k$-го порядка преобразования Chang, а также расщепленной системы, быстрой и медленной подсистем. Расщепляющее преобразование Chang изложено в [9], там же имеются ссылки на некоторые обобщения этого преобразования, указывается важная роль расщепляющих преобразований в теории управления и предложены основанные на декомпозиции параллельные алгоритмы оптимального управления крупномасштабными процессами для линейных и билинейных систем. Обобщение преобразования Chang на системы со многими временными масштабами представлено в $[4,16]$.

Для сингулярно возмущенных систем с запаздыванием расщепляющие преобразования типа Chang строились в $[3,5,8,14]$. В [14] доказано существование непрерывного по малому параметру линейного преобразования координат для частичной декомпозиции сингулярно возмущенной 
системы с распределенным и сосредоточенным запаздыванием, в результате которого в преобразованной системе связь между быстрыми и медленными переменными имеется только через переменные с запаздыванием. Замена переменных типа Chang для линейных стационарных сингулярно возмущенных систем управления с постоянным (не малым) запаздыванием в состоянии, построенное в [3], осуществляется линейным оператором с конечным числом операторов сдвига и приводит исходную сингулярно возмущенную систему с одним запаздыванием в состоянии и без запаздывания в управлении к разделенным подсистемам: медленной со многими запаздываниями в состоянии и управлении и неоднородностью, зависящей от начальных условий, и быстрой с одним запаздыванием в переменной состояния и с неоднородностью.

В [8] построена замена переменных, обобщающая преобразование Chang на линейные стационарные сингулярно возмущенные системы функционально-дифференциальные уравнения с малым сосредоточенным и распределенным запаздыванием в быстрых переменных. Замена расщепляет исходную систему на медленную систему обыкновенных дифференциальных уравнений и быстрые функциональные уравнения. Доказано, что преобразование можно найти в виде асимптотического разложения.

Построенное в настоящей работе невырожденное расщепляющее преобразование обобщает преобразование Chang на линейные стационарные сингулярно возмущенные функционально-дифференциальные системы с конечным запаздыванием в медленной переменной. Доказывается оценка для значений малого параметра, при которых справедлива аппроксимация расщепляющего преобразования.

В отличие от [3] преобразование строится в виде асимптотического ряда, а полученные в результате декомпозиции медленная и быстрая подсистемы не имеют неоднородностей, кроме слагаемых с управлением. В отличие от [14] выполняется полное расщепление системы. В отличие от [5] рассматривается функционально-дифференциальная система с обобщенным типом запаздывания.

2. Постановка задач. Рассматривается следующая линейная стационарная сингулярно возмущенная функционально- дифференциальная система управления с конечным запаздыванием в медленных переменных состояния:

$$
\begin{aligned}
\dot{x}(t) & =\int_{-h}^{0}\left[d A_{1}(\xi)\right] x(t+\xi)+A_{2} y(t)+B_{1} u(t), \quad x \in \mathbb{R}^{n_{1}}, \quad y \in \mathbb{R}^{n_{2}}, \\
\mu \dot{y}(t) & =\int_{-h}^{0}\left[d A_{3}(\xi)\right] x(t+\xi)+A_{4} y(t)+B_{2} u(t), \quad u \in \mathbb{R}^{r}, \quad t \in T=\left[0, t_{1}\right], \\
x(\theta) & =\varphi(\theta), \quad \theta \in[-h, 0], \quad y(0)=y_{0},
\end{aligned}
$$

где $h=\mathrm{const}>0,[-h, 0]$ - интервал запаздывания, $A_{i}(\xi), \xi \in[-h, 0], i=1,3-$ матричные функции ограниченной вариации на $[-h, 0], A_{k}, k=2,4, B_{j}, j=1,2$, - постоянные матрицы подходящих размеров, $\mu$ - малый параметр, $\mu \in\left(0, \mu^{0}\right], \mu^{0} \ll 1, \phi(\theta), \theta \in[-h, 0]$ - непрерывная $n_{1}$-вектор-функция, $u(t)$ - кусочно-непрерывная для $t \geqslant 0 r$-вектор-функция управления, $u(t) \equiv$ $0, t<0, y_{0} \in \mathbb{R}^{n_{2}}$. Пусть $\operatorname{det} A_{4} \neq 0$.

Наличие малого параметра $\mu$ в виде сомножителя при производных части переменных определяет разнотемповый характер изменения фазовых координат $x$ и $y$ в окрестности точки $\mu=0$ : $x$ - медленная переменная, $y$ - быстрая переменная.

С системой (1)-(2) связаны вырожденная система (медленная подсистема) и система пограничного слоя (быстрая подсистема) (см., например, [10]). Вырожденная система для системы (1)-(3) имеет вид

$$
\begin{aligned}
& \dot{x_{s}}(t)=\int_{-h}^{0}\left[d A_{s}(\xi)\right] x_{s}(t+\xi)+B_{s} u_{s}(t), \\
& x_{s}(0)=x_{0}, \quad x_{s}(\theta)=\phi(\theta), \quad \theta \in[-h, 0),
\end{aligned}
$$


где

$$
A_{s}(\xi) \triangleq A_{1}(\xi)-A_{2} A_{4}^{-1} A_{3}(\xi), \quad B_{s} \triangleq B_{1}-A_{2} A_{4}^{-1} B_{2} .
$$

Формально вырожденная система (4) может быть получена из (1)-(2), если положить в ней $\mu=0$ и затем при условии $\operatorname{det} A_{4} \neq 0$ выразить

$$
y_{s}(t)=-A_{4}^{-1}\left[\int_{-h}^{0}\left[d A_{3}(\xi)\right] x_{s}(t+\xi)+B_{2} u_{s}(t)\right]
$$

и исключить переменную $y_{s}(t)$ из уравнения для медленной переменной $x_{s}$.

Система пограничного слоя, соответствующая (1)-(3), получается путем «замораживания» в уравнении (2) для быстрых переменных $y$ медленных переменных $x$ :

$$
\begin{gathered}
\frac{d y_{f}(\tau)}{d \tau}=A_{4} y_{f}(\tau)+B_{2} u_{f}(\tau), \quad \tau=\frac{t}{\mu} \in\left[0, \frac{t_{1}}{\mu}\right], \\
y_{f}(0)=y_{0}-y_{s}(0)=y_{0}+A_{4}^{-1}\left[\int_{-h}^{0}\left[d A_{3}(\xi)\right] \phi(\theta)+B_{2} u_{s}(0)\right] .
\end{gathered}
$$

Обозначим $O(\mu)\left(O\right.$ большое от $\mu$ ) вектор-функцию $f(t, \mu)$ на интервале $\left[t_{1}, t_{2}\right]$ (размерность которой следует из контекста), компоненты $f_{i}(t, \mu)$ которой таковы, что существуют постоянные $\mu^{*}>0, c>0$, такие, что евклидова норма $|f(t, \mu)|$ удовлетворяет неравенству $|f(t, \mu)| \leqslant c \mu$ при всех $\mu \in\left(0, \mu^{*}\right]$ и $t \in\left[t_{1}, t_{2}\right]$.

Задача 1. Построить расщепляющее преобразование, приводящее систему (1)-(3) к двум независимым подсистемам меньшей размерности (отдельно относительно быстрых и медленных переменных), $O(\mu)$-близким к вырожденной системе (4) и системе погранслоя (6).

Задача 2. Доказать, что расщепляющее преобразование может быть построено с любой степенью точности аппроксимации в виде асимптотического разложения по степеням малого параметра, указать итерационную схему нахождения элементов расщепляющего преобразования и получить оценку для значений малого параметра, при которых справедлива аппроксимация расщепляющего преобразования.

3. Невырожденное преобразование. Определим невырожденное преобразование типа Chang (см. [7]) для системы (1)-(2). С этой целью представим систему (1)-(2) в операторной форме.

Пусть $\left(C[a, b], \mathbb{R}^{n}\right)$ - банахово пространство непрерывных функций, отображающих $[a, b]$ в $\mathbb{R}^{n}$ с топологией равномерной сходимости. Для $x \in T \rightarrow \mathbb{R}^{n}$ через $x_{t}:[-h, 0] \rightarrow \mathbb{R}^{n}$ обозначим определенную для $s \in[-h, 0]$ функцию $x_{t}(s)=x(t+s)$. Обозначим $p$ - оператор дифференцирования, $e^{-p \xi}$-оператор сдвига (чистого запаздывания): $e^{-p \xi} x(t)=x(t-\xi)$. Для фиксированного $h \in$ $(0,+\infty)$ обозначим $\mathrm{A}(p)$ непрерывный слева в 0 ограниченный оператор $\mathrm{A}(p):\left(C[-h, 0], \mathbb{R}^{n}\right) \rightarrow \mathbb{R}^{n}$ :

$$
\mathrm{A}(p):=\int_{-h}^{0}[d A(\xi)] e^{p \xi},
$$

где $A(\xi), \xi \in[-h, 0]$ - матричная функция ограниченной вариации на интервале $[-h, 0]$. С учетом $e^{-p \xi} x(t)=x(t-\xi)$ имеем

$$
\mathrm{A}(p) x_{t}=\int_{-h}^{0}[d A(\xi)] x(t+\xi) .
$$

Определим по постоянным матрицам $A_{j}, j=2,4$, из (1)-(2) матричнозначные функции ограниченной вариации на $[-h, 0]$ :

$$
A_{j}(\xi)= \begin{cases}-A_{j}, & \xi<0 \\ 0, & \xi \geqslant 0\end{cases}
$$


и определим операторы $\mathrm{A}_{j}(p), j=2,4$ согласно (8) с использованием (9).

Заметим, что для операторов $\mathrm{A}_{j}(p), j=2,4$ (8) в соответствии с (9) справедливо (см., например, $\left[6\right.$, c. 183]) $\mathrm{A}_{j}(p) y_{t}=y(t)$ и в силу предположения $\operatorname{det} A_{4} \neq 0$ существует обратный оператор $\mathrm{A}_{4}^{-1}(p)$, определяемый по (8), (9) с $A_{4}^{-1}$ вместо $A_{j}$ в $(9)$.

Тогда в соответствии с (8), (9) можно систему (1)-(2) представить в операторной форме:

$$
\begin{aligned}
p x(t) & =\mathrm{A}_{1}(p) x_{t}+\mathrm{A}_{2}(p) y_{t}+B_{1} u(t), \\
\mu p y(t) & =\mathrm{A}_{3}(p) x_{t}+\mathrm{A}_{4}(p) y_{t}+B_{2} u(t), \\
x_{\theta} & =\phi(\theta), \quad y_{\theta}=y_{0}, \quad \theta \leqslant 0 .
\end{aligned}
$$

Введем замену переменных вида:

$$
\left(\begin{array}{l}
x_{t} \\
y_{t}
\end{array}\right)=\mathrm{G}(\mu, p)\left(\begin{array}{c}
\xi_{t} \\
\eta_{t}
\end{array}\right), \quad t \in T
$$

где $\xi_{t}:(-\infty, 0] \rightarrow \mathbb{R}^{n_{1}}, \eta_{t}:(-\infty, 0] \rightarrow \mathbb{R}^{n_{2}}, \xi_{t}=\xi(t+\theta), \eta_{t}=\eta(t+\theta), \theta \leqslant 0, \xi(t) \in \mathbb{R}^{n_{1}}, \eta(t) \in \mathbb{R}^{n_{2}}$, матричный оператор $\mathrm{G}(\mu, p):\left(C(-\infty, 0], \mathbb{R}^{n_{1}+n_{2}}\right) \rightarrow\left(C[-h, 0], \mathbb{R}^{n_{1}+n_{2}}\right)$ имеет вид

$$
\mathrm{G}(\mu, p)=\left(\begin{array}{cc}
E_{n_{1}} & \mu \mathrm{H}(\mu, p) \\
-\mathrm{L}(\mu, p) & E_{n_{2}}-\mu \mathrm{L}(\mu, p) \mathrm{H}(\mu, p)
\end{array}\right) .
$$

$\mathrm{B}(13)$ матричные функции $\mathrm{H}(\mu, p), \mathrm{L}(\mu, p)$ являются решением матричных операторных уравнений: алгебраического уравнения Риккати и уравнения Сильвестра

$$
\begin{gathered}
\mathrm{A}_{3}-\mathrm{A}_{4} \mathrm{~L}+\mu \mathrm{L} A_{1}-\mu \mathrm{LA}_{2} \mathrm{~L}=0, \\
\mu\left(\mathrm{A}_{1}-\mathrm{A}_{2} \mathrm{~L}\right) \mathrm{H}-\mathrm{H}\left(\mathrm{A}_{4}+\mu \mathrm{LA}_{2}\right)+\mathrm{A}_{2}=0 .
\end{gathered}
$$

$\mathrm{C}$ целью сокращения записей здесь и далее, где это не приводит к неоднозначному пониманию, аргументы у матричных операторов $\mathrm{A}_{i}(p), i=1,3, \mathrm{H}(\mu, p), \mathrm{L}(\mu, p)$ будем опускать.

Заметим, что $\operatorname{det} \mathrm{G}(\mu, p) \equiv 1$, значит, преобразование (13) обратимо, причем

$$
\mathrm{G}^{-1}(\mu, p)=\left(\begin{array}{cc}
E_{n_{1}}-\mu \mathrm{HL} & -\mu \mathrm{H} \\
\mathrm{L} & E_{n_{2}}
\end{array}\right), \quad\left(\begin{array}{l}
\xi_{t} \\
\eta_{t}
\end{array}\right)=\mathrm{G}^{-1}(\mu, p)\left(\begin{array}{l}
x_{t} \\
y_{t}
\end{array}\right) .
$$

Определим оператор $\mathrm{A}_{s}(p)$ по $(8)$ с $A_{s}(\xi)(5)$ вместо $A(\xi)$. Очевидно, справедливо:

$$
\mathrm{A}_{s}(p)=\mathrm{A}_{1}(p)-\mathrm{A}_{2}(p) \mathrm{A}_{4}^{-1}(p) \mathrm{A}_{3}(p) .
$$

Лемма 1 (о решении матричных уравнений). Если $\operatorname{det} A_{4} \neq 0$, mо найдется maкое $\mu^{*}>0$, что для всех $\mu \in\left[0, \mu^{*}\right]$ существует непрерывно зависящее от $\mu$ решение $\mathrm{H}(\mu, p), \mathrm{L}(\mu, p)$ системь матричных операторных уравнений (14)-(15) вида

$$
\mathrm{L}(\mu, p)=\mathrm{L}^{0}(p)+\mu \mathrm{L}^{1}(p)+O\left(\mu^{2}\right), \quad \mathrm{H}(\mu, p)=\mathrm{H}^{0}+O(\mu),
$$

¿де

$$
\mathrm{L}^{0}(p)=\mathrm{A}_{4}^{-1}(p) \mathrm{A}_{3}(p), \quad \mathrm{L}^{1}(p)=\mathrm{A}_{4}^{-2}(p) \mathrm{A}_{3}(p) \mathrm{A}_{s}(p), \quad \mathrm{H}^{0}=\mathrm{A}_{2}(p) \mathrm{A}_{4}^{-1}(p) .
$$

Доказательство. Доказательство проведем по схеме, предложенной в [13]. Единственное решение (14) при $\mu=0$

$$
\mathrm{L}^{0}(p) \triangleq \mathrm{L}(0, p)=\mathrm{A}_{4}^{-1}(p) \mathrm{A}_{3}(p) .
$$

Дифференцируя (14) по $\mu$, получаем

$$
\left[A_{4}+\mu \mathrm{L}(\mu, p) \mathrm{A}_{2}(p)\right] \frac{d \mathrm{~L}(\mu, p)}{d \mu}-\mu \frac{d \mathrm{~L}(\mu, p)}{d \mu}\left[\mathrm{A}_{1}(p)-\mathrm{A}_{2}(p) \mathrm{L}(\mu, p)\right]=\mathrm{L}(\mu, p) \mathrm{A}_{1}(p)-\mathrm{L}(\mu, p) \mathrm{A}_{2}(p) \mathrm{L}(\mu, p),
$$

откуда с учетом (18), (19) получаем, что единственное решение этого уравнения при $\mu=0$

$$
\left.\mathrm{L}^{1}(p) \triangleq \frac{d \mathrm{~L}(\mu, p)}{d \mu}\right|_{\mu=0}=\mathrm{A}_{4}^{-1}(p) \mathrm{L}(0, p)\left[\mathrm{A}_{1}(p)-\mathrm{A}_{2}(p) \mathrm{L}(0, p)\right]=\mathrm{A}_{4}^{-2}(p) \mathrm{A}_{3}(p) A_{0}(p) .
$$

Продолжая аналогично, будем получать матричные уравнения, которые решаются последовательно и однозначно в силу $\operatorname{det} A_{4} \neq 0$. Таким образом доказано, что (17) представляет из себя первые два члена разложения в ряд Маклорена по $\mu$ матричного оператора $\mathrm{L}(\mu, p)$. 
Аналогично из (15) при $\mu=0$ имеем единственное решение

$$
\mathrm{H}^{0} \triangleq \mathrm{H}(0, p)=\mathrm{A}_{2}(p) \mathrm{A}_{4}^{-1}(p) .
$$

Дифференцируя (15) по $\mu$, получаем единственное решение

$$
\left.\mathrm{H}^{1}(p) \triangleq \frac{d \mathrm{H}(\mu, p)}{d \mu}\right|_{\mu=0}=\left[\left(\mathrm{A}_{1}(p)-\mathrm{A}_{2}(p) \mathrm{L}(0, p)\right) \mathrm{H}(0, p)-\mathrm{H}(0, p) \mathrm{L}(0, p) \mathrm{A}_{2}(p)\right] \mathrm{A}_{4}^{-1}(p),
$$

и т. д. Продолжая аналогичным образом, будем получать следующие коэффициенты в разложении матричных функций $\mathrm{H}(\mu, p), \mathrm{L}(\mu, p)$. Непрерывная зависимость от $\mu$ доказывается аналогично [14].

Заметим, что $\mathrm{L}(\mu, p)$, определяемое по формуле (17), является единственным в том смысле, что хотя уравнение (14) может иметь несколько решений, но только одно из них аппроксимируется (17).

Лемма 2 (об аппроксимации). Пусть $\operatorname{det} A_{4} \neq 0$. Зависящие от параметра функциональные матрицы $\mathrm{L}(\mu, p), \mathrm{H}(\mu, p)$, являюшиеся решением (14), (15), могут быть найдены с любой степенъю точности в виде

$$
\mathrm{L}(\mu, p)=\sum_{i=0}^{k} \mu^{i} \mathrm{~L}^{i}(p)+O\left(\mu^{k+1}\right), \quad \mathrm{H}(\mu, p)=\sum_{i=0}^{k} \mu^{i} \mathrm{H}^{i}(p)+O\left(\mu^{k}\right),
$$

где $\mathrm{L}^{k}, \mathrm{H}^{k}, k=0,1,2, \ldots$ можно найти согласно итерачионной схеме

$$
\begin{gathered}
\mathrm{L}^{k+1}(p)=A_{4}^{-1}\left(\mathrm{~L}^{k}(p) \mathrm{A}_{1}(p)-\sum_{j=0}^{k} \mathrm{~L}^{k-j}(p) \mathrm{A}_{2}(p) \mathrm{L}^{j}(p)\right), \\
\mathrm{L}^{0}(p)=\mathrm{A}_{4}^{-1}(p) \mathrm{A}_{3}(p), \\
\mathrm{H}^{k+1}(p)=\left(\mathrm{A}_{1}(p) \mathrm{H}^{k}(p)-\mathrm{A}_{2}(p) \sum_{i=0}^{k} \mathrm{~L}^{i}(p) \mathrm{H}^{k-i}(p)-\sum_{i=0}^{k} \mathrm{H}^{i}(p) \mathrm{L}^{k-i}(p) \mathrm{A}_{2}(p)\right) \mathrm{A}_{4}^{-1}(p), \\
\mathrm{H}^{0}(p)=\mathrm{A}_{2}(p) \mathrm{A}_{4}^{-1}(p) .
\end{gathered}
$$

Aппроксимачия (20) имеет место для всех $\mu \in\left[0, \mu^{*}\right)$, где

$$
\mu^{*}=\frac{1}{\left\|\mathrm{~A}_{4}^{-1}\right\|(a+b d+2 b \sqrt{a d / b})}
$$

$u$

$$
a \triangleq\left\|\mathrm{A}_{1}(p)\right\|+\left\|\mathrm{A}_{2}(p)\right\| \cdot\left\|\mathrm{A}_{4}^{-1}(p)\right\| \cdot\left\|\mathrm{A}_{3}(p)\right\|, \quad d \triangleq\left\|\mathrm{A}_{4}^{-1}(p)\right\| \cdot\left\|\mathrm{A}_{3}(p)\right\|, \quad b \triangleq\left\|\mathrm{A}_{2}(p)\right\| .
$$

Доказательство. Доказательство (20)-(22) несложно провести, подставляя разложения (20) в (14), (15) и приравнивая в полученных уравнениях коэффициенты при одинаковых степенях $\mu$. Для доказательства оценки (23) введем

$$
\mathrm{D}(\mu, p)=\mathrm{L}(\mu, p)-\mathrm{L}^{0}(p) .
$$

Используя (21), имеем

$$
\mathrm{A}_{3}(p)=\mathrm{A}_{4}(p) \mathbf{L}^{0}(p) .
$$

Преобразуем (14) (с целью сокращения записей опускаем аргументы $(\mu, p)$ у матричных операторов). Из (24), (25) следуют тождества:

$$
\begin{aligned}
& \mathrm{A}_{3}-\mathrm{A}_{4}\left(\mathrm{D}+\mathrm{L}^{0}\right)+\mu\left(\mathrm{D}+\mathrm{L}^{0}\right) \mathrm{A}_{1}-\mu\left(\mathrm{D}+\mathrm{L}^{0}\right) \mathrm{A}_{2}\left(\mathrm{D}+\mathrm{L}^{0}\right)=0 \Longleftrightarrow \\
& \Longleftrightarrow-\mathrm{A}_{4} \mathrm{D}+\mu\left(\mathrm{D}+\mathrm{L}^{0}\right)\left(\mathrm{A}_{1}-\mathrm{A}_{2}\left(\mathrm{D}+\mathrm{L}^{0}\right)\right)=0 \Longleftrightarrow \\
& \Longleftrightarrow \mathrm{A}_{4} \mathrm{D}=\mu\left(\mathrm{D}+\mathrm{L}^{0}\right)\left(\mathrm{A}_{1}-\mathrm{A}_{2}\left(\mathrm{D}+\mathrm{L}^{0}\right)\right) \Longleftrightarrow \mathrm{A}_{4} \mathrm{D}=\mu\left(\mathrm{D}+\mathrm{L}^{0}\right)\left(\mathrm{A}_{s}-\mathrm{A}_{2} \mathrm{D}\right) \Longleftrightarrow \\
& \Longleftrightarrow\left(\mathrm{L}^{0} \mathrm{~A}_{s}+\mathrm{DA}_{s}-\mathrm{L}^{0} \mathrm{~A}_{2} \mathrm{D}-\mathrm{DA}_{2} \mathrm{D}\right) \triangleq f(\mathrm{D}(\mu, p)) \text {. }
\end{aligned}
$$


Покажем, что для достаточно малых $c=\left\|\mu \mathrm{A}_{4}^{-1}(p)\right\|$ отображение $f(\mathrm{D}(\mu, p))(26)$ является сжимающим отображением.

Пусть $M=\left\{\mathrm{D}:\|\mathrm{D}\| \leqslant(a d / b)^{1 / 2}\right\}$. Для любого $\mathrm{D}, \tilde{\mathrm{D}} \in M$ имеем:

$$
\begin{aligned}
\|f(\mathrm{D})-f(\tilde{\mathrm{D}})\| & =\left\|\mu \mathrm{A}_{4}^{-1}\left((\mathrm{D}-\tilde{\mathrm{D}}) \mathrm{A}_{s}-\mathrm{L}^{0} \mathrm{~A}_{2}(\mathrm{D}-\tilde{\mathrm{D}})-\mathrm{DA}_{2} \mathrm{D}+\tilde{\mathrm{DA}} \mathrm{A}_{2} \tilde{\mathrm{D}} \pm \tilde{\mathrm{D}} \mathrm{A}_{2} \mathrm{D}\right)\right\|= \\
& =\left\|\mu \mathrm{A}_{4}^{-1}\left((\mathrm{D}-\tilde{\mathrm{D}}) \mathrm{A}_{s}-\mathrm{L}^{0} \mathrm{~A}_{2}(\mathrm{D}-\tilde{\mathrm{D}})-(\mathrm{D}-\tilde{\mathrm{D}}) \mathrm{A}_{2} \mathrm{D}-\tilde{\mathrm{D}} \mathrm{A}_{2}(\mathrm{D}-\tilde{\mathrm{D}})\right)\right\| \leqslant \\
& \leqslant\left\|\mu \mathrm{A}_{4}^{-1}\right\| \cdot\|\mathrm{D}-\tilde{\mathrm{D}}\| \cdot\left\|\mathrm{A}_{s}-\mathrm{L}^{0} \mathrm{~A}_{2}-\mathrm{A}_{2} \mathrm{D}-\tilde{\mathrm{DA}} \mathrm{A}_{2}\right\| \leqslant \\
& \leqslant c \cdot\|\mathrm{D}-\tilde{\mathrm{D}}\| \cdot\left(\left\|\mathrm{A}_{s}\right\|+\left\|\mathrm{L}^{0}\right\| \cdot\left\|\mathrm{A}_{2}\right\|+\left\|\mathrm{A}_{2}\right\| \cdot\|\mathrm{D}\|+\|\tilde{\mathrm{D}}\| \cdot\left\|\mathrm{A}_{2}\right\|\right) \leqslant \\
& \leqslant c \cdot\|\mathrm{D}-\tilde{\mathrm{D}}\| \cdot\left(a+d b+2 b(a d / b)^{1 / 2}\right) \leqslant\|\mathrm{D}-\tilde{\mathrm{D}}\|,
\end{aligned}
$$

если

$$
c<\frac{1}{a+d b+2 b(a d / b)^{1 / 2}} .
$$

Здесь используем очевидные соотношения:

$$
\left\|\mathrm{A}_{s}(p)\right\| \stackrel{(5)}{=} \leqslant\left\|\mathrm{A}_{1}(p)\right\|+\left\|\mathrm{A}_{2}(p)\right\| \cdot\left\|\mathrm{A}_{4}^{-1}(p)\right\| \cdot\left\|\mathrm{A}_{3}\right\| \triangleq a, \quad\left\|\mathrm{~L}^{0}(p)\right\| \stackrel{(18)}{=}\left\|\mathrm{A}_{4}^{-1}(p)\right\| \cdot\left\|\mathrm{A}_{3}(p)\right\| \triangleq d .
$$

Таким образом, в силу принципа сжимающих отображений уравнение (26) имеет единственное решение $\mathrm{L}$, которое может быть найдено с любой степенью точности, начиная с любого $\mathrm{D}_{0} \in M$. Граница (23) для $\mu$ следует из (27).

Используя матричные параметры системы (1)-(2) и матричные функции $\mathrm{L}(\mu, p), \mathrm{H}(\mu, p)$, определим зависящие от параметра матричные функции

$$
\begin{aligned}
& \mathrm{A}_{\xi}(\mu, p) \triangleq \mathrm{A}_{1}(p)-\mathrm{A}_{2}(p) \mathrm{L}(\mu, p), \\
& \mathrm{B}_{\xi}(\mu, p) \triangleq B_{1}-\mathrm{H}(\mu, p) B_{2}-\mu \mathrm{H}(\mu, p) \mathrm{L}(\mu, p) B_{1}, \\
& \mathrm{~A}_{\eta}(\mu, p) \triangleq \mathrm{A}_{4}(p)+\mu \mathrm{L}(\mu, p) \mathrm{A}_{2}(p), \\
& \mathrm{B}_{\eta}(\mu, p) \triangleq B_{2}+\mu \mathrm{L}(\mu, p) B_{1} .
\end{aligned}
$$

Из (28), (5), (18) имеем

$$
\begin{array}{ll}
\mathrm{A}_{\xi}(\mu, p)=\mathrm{A}_{s}(p)+O(\mu), & \mathrm{B}_{\xi}(\mu, p)=B_{s}+O(\mu), \\
\mathrm{A}_{\eta}(\mu, p)=A_{4}+O(\mu), & \mathrm{B}_{\eta}(\mu, p)=B_{2}+O(\mu) .
\end{array}
$$

Теорема 1 (о расщепляющем преобразовании). Если $\operatorname{det} A_{4} \neq 0$, то найдется такое $\mu^{*}>0$, что в результате замены переменных (12), (13) для всех $\mu \in\left(0, \mu^{*}\right]$ система (1)-(3) преобразуется в эквивалентную систему с разделенными движениями

$$
\begin{gathered}
\dot{\xi}(t)=\left(\mathrm{A}_{1}(p)-\mathrm{A}_{2}(p) \mathrm{L}(p)\right) \xi_{t}+\left(B_{1}-\mathrm{H}(p) B_{2}-\mu \mathrm{H}(p) \mathrm{L}(p) B_{1}\right) u_{t}, \\
\mu \dot{\eta}(t)=\left(\mathrm{A}_{4}(p)+\mu \mathrm{L}(p) \mathrm{A}_{2}(p)\right) \eta_{t}+\left(B_{2}+\mu \mathrm{L}(p) B_{1}\right) u_{t}, \quad t \in T, \\
\xi_{\theta}=\left(E_{n_{1}}-\mu \mathrm{H}(p) \mathrm{L}(p)\right) x_{\theta}-\mu \mathrm{H}(p) y_{\theta}, \\
\eta_{\theta}=\mathrm{L}(p) x_{\theta}+y_{\theta}, \quad \theta \leqslant 0 .
\end{gathered}
$$

Доказательство. Для обоснования декомпозиции исходной системы (1)-(2) введем при фиксированном $\mu>0$ матричные функции

$$
\mathrm{A}(\mu, p)=\left(\begin{array}{cc}
\mathrm{A}_{1}(p) & \mathrm{A}_{2}(p) \\
\mathrm{A}_{3}(p) / \mu & \mathrm{A}_{4}(p) / \mu
\end{array}\right), \quad B(\mu)=\left(\begin{array}{c}
B_{1} \\
B_{2} / \mu
\end{array}\right),
$$

и обозначим $z(t)=\{x(t), y(t)\}^{\prime}$. Тогда систему (10) можно записать в виде

$$
p z(t)=\mathrm{A}(\mu, p) z_{t}+B(\mu) u(t) .
$$

с начальными условиями

$$
z_{\theta}=\left\{x_{\theta}, y_{\theta}\right\}^{\prime} .
$$


Применим к системе (32) преобразование (12), (13) и умножим преобразованное уравнение слева на $\operatorname{diag}\left\{E_{n_{1}}, \mu E_{n_{2}}\right\} \mathrm{G}^{-1}(p)$. В результате получим эквивалентную (32) систему

$$
p \operatorname{diag}\left\{E_{n_{1}}, \mu E_{n_{2}}\right\} \zeta(t)=A_{\xi \eta}(\mu, p) \zeta_{t}+B_{\xi \eta}(\mu, p) u_{t},
$$

где $\zeta(t)=\{\xi(t), \eta(t)\}^{\prime}$

$$
\begin{aligned}
& A_{\xi \eta}(\mu, p)=\mathrm{G}^{-1}(\mu, p) A(\mu, p) \mathrm{G}(\mu, p)=\left(\begin{array}{cc}
A_{\xi}(\mu, p) & 0_{n_{1} \times n_{2}} \\
0_{n_{2} \times n_{1}} & A_{\eta}(\mu, p)
\end{array}\right), \\
& B_{\xi \eta}(\mu, p)=\mathrm{G}^{-1}(\mu, p) B(\mu)=\left(\begin{array}{c}
B_{\xi}(\mu, p) \\
B_{\eta}(\mu, p)
\end{array}\right) .
\end{aligned}
$$

Систему (34) с учетом (35) можно представить как систему с разделенными движениями

$$
\begin{aligned}
p \xi(t) & =A_{\xi}(\mu, p) \xi_{t}+B_{\xi}(\mu, p) u_{t}, \\
\mu p \eta(t) & =A_{\eta}(\mu, p) \eta_{t}+B_{\eta}(\mu, p) u_{t},
\end{aligned}
$$

что в силу (28) равносильно (30).

Применение преобразования (12), (13) к начальным условиям (33) дает начальные условия (31) для расщепленной системы (30).

Из (28)-(30) следует, что вырожденная система (4) и система погранслоя (6) представляют из себя асимптотическую декомпозицию исходной системы (1)-(2).

Основываясь на итерационном решении $k$-го порядка $(k=0,1, \ldots)$ из $(20)$, несложно по (13) определить аппроксимацию преобразования $\mathrm{G}(\mu, p) k$-го порядка, а также аппроксимацию $k$-го порядка быстрой и медленной подсистем расщепленной системы (30). В отличие от системы (36), которая в общем случае представляет систему с бесконечным запаздыванием, при каждом фиксированном $k(k=0,1, \ldots)$ аппроксимация $k$-го порядка расщепленной системы $(30)$ будет системой с конечным запаздыванием.

4. Приложения расщепляющего преобразования. Построенное расщепляющее преобразование можно использовать для обоснования разделения спектра и анализа устойчивости системы (1)-(2), (3), решения задач управления системой (1)-(2) с помощью композитных регуляторов, сконструированных на основе регуляторов отдельно для быстрой и медленной подсистем.

Обоснуем разделение спектра. Пусть $\mathbb{C}$ - поле комплексных чисел. Обозначим через

$$
W(\mu, \lambda)=\operatorname{det}\left[\lambda E_{n_{1}+n_{2}}-\mathrm{A}(\mu, \lambda)\right], \quad \lambda \in \mathbb{C},
$$

матричную характеристическую функцию системы (1)-(2), через $\sigma(\mu)=\{\lambda \in \mathbb{C}: W(\lambda, \mu)=0\}-$ спектр (множество собственных значений) системы, через

$$
W_{s}(\lambda)=\operatorname{det}\left[\lambda E_{n_{1}}-\mathrm{A}_{s}(\lambda)\right], \quad W_{f}(\lambda)=\operatorname{det}\left[\lambda E_{n_{2}}-A_{4}\right]
$$

- характеристическую функцию вырожденной системы (4) и характеристический полином системы погранслоя (6), соответственно, и через $\sigma_{s}=\left\{\lambda \in C: W_{s}(\lambda)=0\right\}$ и $\sigma_{f}=\left\{\lambda \in C: W_{f}(\lambda)=\right.$ $0\}$ - спектры этих систем.

Пусть выполнено условие $\operatorname{det} A_{4} \neq 0$.

Теорема 2 (о разделении спектра). Спектр системы (1)-(2) разделяется на «быструю» часть, в которой $n_{2}$ корней характеристического уравнения $W(\mu, \lambda)=0$ имеют вид

$$
\frac{1}{\mu} \lambda_{i}(\mu), \quad \lim _{\mu \rightarrow 0} \lambda_{i}(\mu)=\lambda_{f i},
$$

где $\lambda_{f i} \in \sigma_{f}, u$ «медленную», элементы которой при $\mu \rightarrow 0$ стремятся $\kappa$ элементам спектра вырожденной системы (4):

$$
\lim _{\mu \rightarrow 0} \lambda_{i}(\mu)=\lambda_{s i}, \quad \lambda_{s i} \in \sigma_{s}
$$


Доказательство. Из теоремы 1 следует, что в результате применения преобразования (12), (13) система (1)-(2) переходит в эквивалентную ей систему (30), которая с учетом (5), (28), (36), (17)-(18) в операторной форме может быть представлена в виде:

$$
\begin{aligned}
p \xi(t) & =\left(\mathrm{A}_{s}(p)+O(\mu)\right) \xi_{t}+\left(B_{s}+O(\mu)\right) u(t), \quad \xi \in \mathbb{R}^{n_{1}}, \quad u \in \mathbb{R}^{r}, \\
\mu p \eta(t) & =\left(A_{4}+O(\mu)\right) \eta(t)+\left(B_{2}+O(\mu)\right) u(t), \quad \eta \in \mathbb{R}^{n_{2}}, \quad t \in T=\left[0, t_{1}\right] .
\end{aligned}
$$

Заметим, что подсистема (39) $O(\mu)$-близка к вырожденной системе (4), а (40) - к системе погранслоя (6).

Рассмотрим характеристическое уравнение системы (1)-(2):

$$
W(\mu, \lambda)=0
$$

и характеристическое уравнение эквивалентной ему системы (39)-(40):

которое очевидно равносильно

$$
\operatorname{det}\left(\begin{array}{cc}
\lambda E_{n_{1}}-\mathrm{A}_{s}(\mu, \lambda)+O(\mu) & 0_{n_{1} \times n_{2}} \\
0_{n_{2} \times n_{1}} & \lambda E_{n_{2}}-A_{4}+O(\mu)
\end{array}\right)=0
$$

$$
\operatorname{det}\left[\lambda E_{n_{1}}-A_{s}(\mu, \lambda)+O(\mu)\right] \cdot \operatorname{det}\left[\lambda E_{n_{2}}-A_{4}+O(\mu)\right]=0 .
$$

Сравнивая последнее уравнение с характеристическими уравнениями подсистем, приходим к заключению о справедливости утверждения теоремы 2.

Таким образом установлено, что система (1)-(2) может быть аппроксимативно разделена на медленную подсистему (39) с «маленькими» собственными значениями и быструю подсистему (40) с «большими» собственными значениями. При этом медленная и быстрая подсистемы (39), (40) $O(\mu)$-близки к вырожденной системе (4) и системе погранслоя (6), соответственно. Характеристическое уравнение исходной системы (41) может анализироваться асимптотически через два намного более простых, не зависящих от малого параметра, уравнения квазимногочленного и многочленного типа (38).

Разделение спектра на два непересекающихся множества для сингулярно возмущенных систем без запаздывания доказывается на основании декомпозиции в [13], для сингулярно возмущенной линейной стационарной системы с малыми запаздываниями в состоянии и управляющих переменных обосновано в [8], для сингулярно возмущенных систем с одним постоянным запаздыванием в медленной переменной - в [5], для сингулярно возмущенных систем с запаздываниями двух масштабов - постоянным в медленных переменных и малым (порядка малости $\mu$ ) в быстрых переменных - в $[11]$. В $[5,8,13]$ доказательство основано на расщепляющем преобразовании типа Chang.

\section{5. Примеры.}

Пример 1. Рассмотрим следующую систему:

$$
\begin{aligned}
\dot{x}(t) & =-x(t)-y(t), \quad x \in \mathbb{R}, \quad y \in \mathbb{R}, \quad t \in[0,4], \\
\mu \dot{y} & =k x(t-2)-y(t)+u(t), \quad u \in \mathbb{R}, \quad k \in \mathbb{R}, \\
x(\theta) & =\phi(\theta) \equiv 1, \quad \theta \in[-2,0], \quad y(0)=y_{0}=3 .
\end{aligned}
$$

Система (42) является частным случаем система (1)-(3) с параметрами $h=2, t_{0}=0, t_{1}=4$, и скалярными функциями:

$$
\begin{gathered}
A_{1}(\xi)= \begin{cases}1, & \xi \leqslant-2, \\
1, & -2<\xi<0, \quad A_{2}=-1, \\
0, & \xi \geqslant 0,\end{cases} \\
A_{3}(\xi)= \begin{cases}-k, & \xi \leqslant-2, \\
0, & -2<\xi<0, \quad A_{4}=-1, \quad B_{1}=0, \quad B_{2}=1 . \\
0, & \xi \geqslant 0,\end{cases}
\end{gathered}
$$


Для построения расщепляющего преобразования (12), (13) согласно (18) имеем первые члены рядов (20) для системы (42):

$$
\begin{gathered}
\mathrm{H}^{0}=1, \quad \mathrm{H}^{1}=-1, \quad \mathrm{~L}^{i}(p)=\int_{-2}^{0}\left[d L^{i}(\xi)\right] e^{p \xi}, \quad i=0,1, \\
L^{0}(\xi)=\left\{\begin{array}{ll}
k, & \xi \leqslant-4, \\
k, & -4<\xi \leqslant-2, \\
0, & -2<\xi<0, \\
0, & \xi \geqslant 0,
\end{array} \quad L^{1}(\xi)= \begin{cases}k-k^{2}, & \xi \leqslant-4, \\
k, & -4<\xi \leqslant-2, \\
0, & -2<\xi<0, \\
0, & \xi \geqslant 0 .\end{cases} \right.
\end{gathered}
$$

В соответствии с (5), (43) имеем параметры вырожденной системы (4):

$$
\mathrm{A}_{s}(p)=\int_{-2}^{0}\left[d A_{s}(\xi)\right] e^{p \xi}, \quad B_{s}(p)=-1, \quad A_{s}(\xi)= \begin{cases}k+1, & \xi \leqslant-2, \\ 1, & -2<\xi<0 \\ 0, & \xi \geqslant 0 .\end{cases}
$$

Окончательно имеем вырожденную систему (4) для системы (42):

$$
\begin{aligned}
& \dot{x}_{s}(t)=-x_{s}(t)-k x_{s}(t-2)-u_{s}(t), \quad t \in[0,4], \\
& x_{s}(\theta)=1, \quad \theta \in[-2,0],
\end{aligned}
$$

и систему погранслоя:

$$
\begin{aligned}
\frac{d}{d \tau} y_{f}(\tau) & =-y_{f}(\tau)+u_{f}(\tau), \quad \tau \in\left[0, \frac{4}{\mu}\right], \\
y_{f}(0) & =3-k-u_{s}(0),
\end{aligned}
$$

которые совпадают с аппроксимацией 0-го порядка расщепленной системы.

Аппроксимация 1-го порядка расщепленной системы имеет вид:

$$
\begin{gathered}
\dot{\xi}(t)=-\xi(t)-(k+\mu k) \xi(t-2)-\mu k \xi(t-4)+(-1+\mu) u(t), \quad t \in[0,4], \\
\frac{d}{d \tau} \eta(\tau)=-\eta(\tau)+\mu k \eta\left(\tau-\frac{2}{\mu}\right)+u(\tau), \quad \tau \in\left[0, \frac{4}{\mu}\right] \\
\xi_{\theta}=1-3 \mu, \quad \eta_{\theta}=3-k-\mu, \quad \theta \in[-2,0] .
\end{gathered}
$$

Заметим, что с учетом вида (45) функций, участвующих в определении операторов (44), операторы $\mathrm{L}^{0}(p), \mathrm{L}^{1}(p), \mathrm{A}_{s}(p)$ можно представить в виде:

$$
\mathrm{L}^{0}(p)=-k e^{-2 p}, \quad \mathrm{~L}^{1}(p)=-k e^{-2 p}\left(1+e^{-2 p}\right), \quad A_{s}(p)=-1-k e^{-2 p} .
$$

Аппроксимация (20) для (42) действительна для всех $\mu \in\left[0, \mu^{*}\right)$, где $\mu^{*}=(1+2 k+$ $2 \sqrt{(1+k) k})^{-1}$.

Пример 2. Рассмотрим систему:

$$
\begin{aligned}
\dot{x}_{1}(t) & =-x_{1}(t)+2 x_{2}(t)-x_{2}(t-1)-y(t), \\
\dot{x}_{2}(t) & =-x_{1}(t)+x_{1}(t-1)+2 x_{2}(t)-x_{2}(t-1), \\
\mu \dot{y} & =-x_{1}(t-1)-y(t)+u(t), \quad t \in[0,2], \\
x(\theta) & =\phi(\theta)=\theta, \quad \theta \in[-2,0], \quad y(0)=y_{0}=5 .
\end{aligned}
$$


Система (48) является частным случаем системы (1)-(2) с параметрами $n_{1}=2, n_{2}=r=1$, $l=1, h=1$ и матричными функциями

$$
\begin{aligned}
& A_{1}(\xi)= \begin{cases}-A_{10}-A_{11}, & \xi \leqslant-1, \\
-A_{10}, & -1<\xi<0, \quad A_{2}=\left(\begin{array}{r}
-1 \\
0
\end{array}\right), \\
0_{2 \times 2}, & \xi \geqslant 0,\end{cases} \\
& A_{3}(\xi)= \begin{cases}-A_{30}-A_{31}, & \xi \leqslant-1, \\
0_{1 \times 2}, & -1<\xi<0, \quad A_{4}=-1, \\
0_{1 \times 2}, & \xi \geqslant 0,\end{cases} \\
& A_{10}=\left(\begin{array}{ll}
-1 & 2 \\
-1 & 2
\end{array}\right), \quad A_{11}=\left(\begin{array}{ll}
0 & -1 \\
1 & -1
\end{array}\right), \quad B_{1}=\left(\begin{array}{l}
0 \\
0
\end{array}\right) \text {, } \\
& A_{30}=\left(\begin{array}{ll}
0 & 0
\end{array}\right), \quad A_{31}=\left(\begin{array}{ll}
-1 & 0
\end{array}\right), \quad B_{2}=(1) .
\end{aligned}
$$

Для построения расщепляющего преобразования (12), (13) согласно (18) имеем первые члены рядов (20) для системы (48):

$$
\begin{aligned}
& \mathrm{H}^{0}=\left(\begin{array}{l}
1 \\
0
\end{array}\right), \quad \mathrm{H}^{1}=\left(\begin{array}{c}
1 \\
1-e^{-p}
\end{array}\right), \quad \mathrm{L}^{i}(p)=\int_{-1}^{0}\left[d L^{i}(\xi)\right] e^{p \xi}, \quad i=0,1, \\
& L^{0}(\xi)=\left\{\begin{array}{ll}
A_{30}+A_{31}, & \xi \leqslant-1, \\
0_{1 \times 2}, & -1<\xi<0, \\
0_{1 \times 2}, & \xi \geqslant 0,
\end{array} \quad L^{1}(\xi)= \begin{cases}(0,-1), & \xi \leqslant-1, \\
(0,0), & \xi>-1 .\end{cases} \right.
\end{aligned}
$$

В соответствии с (5), (49) параметры медленной подсистемы:

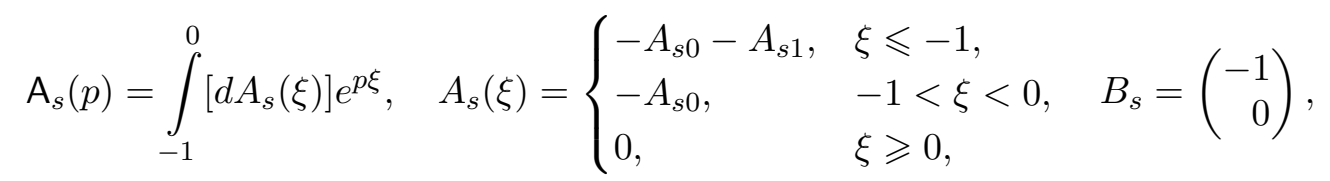

где

$$
A_{s 0}=\left(\begin{array}{ll}
-1 & 2 \\
-1 & 2
\end{array}\right), \quad A_{s 1}=\left(\begin{array}{ll}
1 & -1 \\
1 & -1
\end{array}\right) .
$$

Окончательно для системы (48) имеем вырожденную систему (4):

$$
\begin{aligned}
& \dot{x}_{s 1}(t)=-x_{s 1}(t)+x_{s 1}(t-1)+2 x_{s 2}(t)-x_{s 2}(t-1)-u_{s}(t), \\
& \dot{x}_{s 2}(t)=-x_{s 1}(t)+x_{s 1}(t-1)+2 x_{s 2}(t)-x_{s 2}(t-1),
\end{aligned}
$$

и систему погранслоя (6):

$$
\frac{d y_{f}(\tau)}{d \tau}=-y_{f}(\tau)+u_{f}(\tau)
$$

Заметим, что операторы для подсистем можно записать в виде:

$$
\mathrm{L}^{0}(p)=\left(e^{-p}, 0\right), \quad \mathrm{L}^{1}(p)=\left(0, e^{-p}\right), \quad A_{s}(p)=\left(\begin{array}{ll}
-1+e^{-p} & 2-e^{-p} \\
-1+e^{-p} & 2-e^{-p}
\end{array}\right) .
$$

Характеристическая функция (37) для системы (48) имеет вид:

$$
W(\mu, \lambda)=\frac{1}{\mu}\left(\lambda(\lambda-1)(1+\mu \lambda)-\mu \lambda e^{-\lambda}\left(2-e^{-\lambda}-\lambda\right)\right),
$$

спектр системы (48):

$$
\sigma(\mu)=\left\{0,1+O(\mu),-\frac{1}{\mu}+O(\mu)\right\} .
$$

Характеристические функции (38) для вырожденной системы (51) и системы погранслоя (52):

$$
W_{s}(\lambda)=\lambda^{2}-\lambda+2 e^{-\lambda}\left(1-e^{-\lambda}\right), \quad W_{f}(\lambda)=\lambda+1 .
$$


Спектр вырожденной системы (51) и системы погранслоя (52) для (48): $\sigma_{s}=\{0,1\}, \sigma_{f}=\{-1\}$, что с учетом (2) иллюстрирует вторую часть теоремы 1.

Пример 3. Рассмотрим систему

$$
\begin{aligned}
\dot{x}(t) & =-x(t)+u(t), \\
\mu \dot{y}(t) & =-y(t)+\int_{-1}^{0} e^{\xi} x(t+\xi) d \xi, \quad t \in[0,2], \\
x(\theta) & =\phi(\theta)=\theta, \quad \theta \in[-1,0], \quad y(0)=y_{0}=7 .
\end{aligned}
$$

Система (53) является частным случаем системы (1)-(2) с параметрами $n_{1}=1, n_{2}=r=1$, $l=1, h=1$ и матричными функциями

$$
A_{1}(\xi)\left\{\begin{array}{ll}
1, & \xi<0, \\
0, & \xi \geqslant 0,
\end{array} \quad A_{2}=0, \quad A_{3}(\xi)=e^{\xi}, \quad A_{4}(\xi)=\left\{\begin{array}{ll}
1, & \xi<0, \\
0, & \xi \geqslant 0,
\end{array} \quad B_{1}=1, \quad B_{2}=0 .\right.\right.
$$

Для построения расщепляющего преобразования (12), (13) согласно (18) имеем первые члены рядов (20) для системы (53):

$$
\begin{gathered}
\mathrm{H}^{0}=0, \quad \mathrm{H}^{1}=0, \quad \mathrm{~L}^{i}(p)=\int_{-1}^{0}\left[d L^{i}(\xi)\right] e^{p \xi}, \quad i=0,1, \\
L^{0}(\xi)=e^{-\xi}, \quad \xi \in[-h, 0], \quad L^{1}(\xi)= \begin{cases}e^{\xi}, & \xi<0, \\
0, & \xi \geqslant 0 .\end{cases}
\end{gathered}
$$

В соответствии с (5), (53) имеем параметры вырожденной системы:

$$
\mathrm{A}_{s}(p)=\int_{-1}^{0}\left[d A_{s}(\xi)\right] e^{p \xi}, \quad A_{s}(\xi)=\left\{\begin{array}{ll}
1, & \xi<0, \\
0, & \xi \geqslant 0,
\end{array} \quad B_{s}(p)=1\right.
$$

Окончательно для (53) имеем вырожденную систему

$$
\dot{x}_{s}(t)=-x_{s}(t)+u_{s}(t), \quad x_{s}(0)=0,
$$

и систему погранслоя

$$
\frac{d y_{f}(\tau)}{d \tau}=-y_{f}(\tau), \quad y_{f}(0)=7 .
$$

Аппроксимация (20) для (53) действительна для всех $\mu \in[0,1)$.

Характеристическая функция (37) для системы (53) имеет вид:

$$
W(\mu, \lambda)=(\lambda+1)\left(\lambda+\frac{1}{\mu}\right)
$$

поэтому система (53) имеет конечный спектр:

$$
\sigma(\mu)=\{-1,-1 / \mu\} .
$$

Характеристические функции для вырожденной системы (3) и (3):

$$
w_{s}(\lambda)=(1+\lambda), \quad w_{f}(\lambda)=(1+\lambda) .
$$

Спектры вырожденной системы (3) и системы погранслоя (3): $\sigma_{s}=\{-1\}, \sigma_{f}=\{-1\}$, что соответствует утверждению теоремы 1. 
6. Заключение. В статье рассматривается линейная стационарная сингулярно возмущенная функционально-дифференциальная система управления с запаздыванием в медленных переменных состояния. Обосновано построение невырожденного преобразования типа Chang переменных исходной системы, которое разделяет исходную сингулярно возмущенную систему на две несвязанные $O(\mu)$-близкие к независящим от $\mu$ подсистемы - медленную и быструю подсистемы меньших размеров, чем исходные.

Расщепляющее преобразование может быть построено с любой степенью точности в виде асимптотического разложения по степеням малого параметра. Получена оценка значений параметра, при которой справедлива аппроксимация. В соответствии с формулами (21), (22) алгоритм декомпозиции может быть реализован в виде программ для систем компьютерной алгебры. На основании декомпозиции обосновано разделение спектра системы на два непересекающихся множества: отдельно с «маленькими» и «большими» собственными значениями.

Применение построенного невырожденного преобразования позволяет понизить порядок рассматриваемой функционально-дифференциальной многомерной сингулярно возмущенной системы и свести решение ряда задач устойчивости, управления и оценивания для больших систем с сингулярными возмущениями к системам меньшей размерности, не зависящим или регулярно зависящим от малого параметра. Такой подход продемонстрирован в [5] на примере решения задачи управления медленной частью спектра линейным динамическим регулятором по медленным переменным.

Использование в расщепленной системе (30) аппроксимаций (20) позволяет получать последовательность систем уравнений с разделенными движениями, обеспечивающих требуемую точность собственных значений, решений $x(t), y(t)$ системы (1)-(2). Полученные результаты можно использовать для решения задач анализа и синтеза линейных стационарных сингулярно возмущенных функционально-дифференциальных систем управления запаздывающего типа.

\section{СПИСОК ЛИТЕРАТУРЫ}

1. Васильева А. Б., Дмитриев М. Г. Сингулярные возмущения в задачах оптимального управления// Итоги науки и техн. Сер. Мат. анал. - 1982. - 20. - С. 3-77.

2. Дмитриев М. Г., Курина Г. А. Сингулярные возмущения в задачах управления// Автомат. телемех. - 2006. - 1. - C. 3-51.

3. Копейкина Т. Б. Об управляемости линейных сингулярно возмущенных систем с запаздыванием// Диффер. уравн. - 1989. - С. 1508-1518.

4. Курина Г. А. О полной управляемости разнотемповых сингулярно возмущенных систем// Мат. заметки. - 1992. - 52, № 4. - С. 56-61.

5. Цехан О. Б. Расщепляющее преобразование для линейной стационарной сингулярно возмущенной системы с запаздыванием и его применение к анализу и управлению спектром// Весн. ГрДУ ім. Я. Купалы. Сер 2. Мат. - 2017. - 7, № 1. - С. 50-61.

6. Хейл Дж. Теория функционально-дифференциальных уравнений. - М.: Мир, 1984.

7. Chang K. Singular perturbations of a general boundary value problem// SIAM J. Math. Anal. — 1972. — 3, № 3. - P. 520-526.

8. Fridman E. Decoupling transformation of singularly perturbed systems with small delays and its applications// Z. Angew. Math. Mech. Berlin. - 1996. - 76, № 2. - P. 201-204.

9. Gajic Z., Shen X. Parallel algorithms for optimal control of large scale linear systems. - London: SpringerVerlag, 1993.

10. Glizer $V$. Ya. L2-Stabilizability conditions for a class of nonstandard singularly perturbed functionaldifferential systems// Dyn. Cont. Discr. Impuls. Systems. Ser. es B: Appl. Alg. — 2009. — 16. — P. 181-213.

11. Glizer V. Ya. Approximate state-space controllability of linear singularly perturbed systems with two scales of state delays// Asympt. Anal. - 2018. — 107, № 1-2. - P. 73-114.

12. Kokotovic P. V., Haddad A. H. Controllability and time-optimal control of systems with slow and fast modes// IEEE Trans. Automat. Contr. — 1975. - 20, № 1. - P. 111-113.

13. Kokotovic P. V., Khalil H. K., O'Reilly J. Singular Perturbations Methods in Control: Analysis and Design. — New York: Academic Press, 1986. 
14. Magalhaes L. T. Exponential estimates for singularly perturbed linear functional differential equations// J. Math. Anal. Appl. — 1984. - 103. - P. 443-460.

15. Pekar L., Gao Q. Spectrum analysis of LTI continuous-time systems with constant delays: a literature overview of some recent results// IEEE Acc. — 2018. - 6, № 1. - P. 35457-35491.

16. Prljaca N., Gajic Z. General transformation for block diagonalization of multitime-scale singularly perturbed linear systems// IEEE Trans. Automat. Contr. — 2008. — 53, № 5. — P. 1303-1305.

17. Yang X., Zhu J. J. A generalization of Chang transformation for linear time-varying systems// Proc. IEEE Conf. on Decision and Control. - Atlanta, GA, 2010. — P. 6863-6869.

18. Yang X., Zhu J. J. Chang transformation for decoupling of singularly perturbed linear slowly time-varying systems// Proc. 51st IEEE Conf. on Decision and Control (December 10-13, Maui, Hawaii, USA), 2012. P. $5755-5760$.

19. Zhang Y., Naidu D. S., Cai C, et al. Singular perturbations and time scales in control theories and applications: An overview 2002 - 2012// Int. J. Inf. Syst. Sci. — 2014. — 9, № 1. — P. 1-36.

Цехан Ольга Борисовна

Гродненский государственный университет им. Янки Купалы, Гродно, Республика Беларусь E-mail: tsekhan@grsu.by 